\title{
AAV delivery of shRNA against IRS1 in GABAergic neurons in rat hippocampus impairs spatial memory in females and male rats
}

\author{
Authors \\ Sandra Sánchez-Sarasúa ${ }^{1}$, Alberto Ribes-Navarro ${ }^{1,2}$, María Teresa Beltrán-Bretones ${ }^{1}$, Ana María Sánchez- \\ Pérez $^{1 *}$ \\ ${ }^{1}$ Department of Medicine. Universitat Jaume I. Castellon (Spain) \\ ${ }^{2}$ Present address: Instituto de Acuicultura de Torre de la Sal (IATS-CSIC), 12595 Ribera de Cabanes, \\ Castellón, Spain \\ *Corresponding author; Ana María Sánchez-Pérez; sanchean@uji.es;
}

ORCID ID 0000-0002-5811-0005; tel. 349643859

\begin{abstract}
Brain insulin resistance is a major factor leading to impaired cognitive function and it is considered at the onset of Alzheimer's disease. Insulin resistance is intimately linked to inflammatory conditions, many studies have revealed how pro-inflammatory cytokines lead to insulin resistance, by inhibiting IRS1 function. Thus, the dysfunction of insulin signaling is concomitant with inflammatory biomarkers. However, the specific effect of IRS1 impaired function in otherwise healthy brain has not been dissected out. So, we decided in our study, to study the specific role of IRS1 in the hippocampus, in the absence of comorbidities. To that end, shRNA against rat and human IRS1 was designed and tested in cultured HEK cells to evaluate mRNA levels and specificity. The best candidate sequence was encapsulated in an AAV vector (strain DJ8) under the control of the cytomegalovirus promoter and together with the green fluorescent protein gene as a reporter. AAV-CMV-shIRS1-EGFP and control AAV-CMV-EGFP were inoculated into the dorsal hippocampus of females and males' Wistar rats. One month later, animals undertook a battery of behavioral paradigms evaluating spatial and social memory and anxiety. Our results suggest that females displayed increased susceptibility to AAV-shIRS1 in the novel recognition object paradigm; whereas both females and males show impaired performance in the T-maze when infected with AAV-shIRS1 compared to control. Anxiety parameters were not affected by AAV-shIRS1 infection. We observed specific fluorescence within the hilum of the dentate gyrus, in immunocharacterized parvalbumin and somatostatin neurons. AAV DJ8 did not enter astrocytes. Intense green fibers were found in the fornix, mammillary bodies, and in the medial septum indicating that hippocampal efferent had been efficiently targeted by the AAV DJ8 infection. We observed that AAV-shIRS1 reduced significantly synaptophysin labeling in hippocampal-septal projections compared to controls. These results support that, small alterations in the insulin/IGF1 pathway in specific hippocampal circuitries can underlie alterations in synaptic plasticity and affect behavior, in the absence of inflammatory conditions
\end{abstract}

Keywords: Insulin resistance; IRS2; hippocampal dentate gyrus; social memory; synaptophysin; immunofluorescence; synaptic plasticity. 


\section{Introduction}

Insulin and IGF1 actions are mediated by the insulin and IGF1 receptors that can activate similar signaling pathways with pleiotropic actions in mammals. In the brain, both peptides have been shown to modulate cognitive function (Al-Delaimy et al. 2009; Cholerton et al. 2013). The signaling cascades elicited by insulin and IGF1 are almost identical and both mediated by the scaffolding proteins, the insulin receptor substrates (IRS) (Kleinridders et al. 2014; Bedse et al. 2015). There are four IRSs known isoforms, being the IRS1 and IRS2 the most abundant and studied, up to date (Bedse et al. 2015). In spite the fact that both, IRS1 and 2 have been shown to mediate insulin and IGF1 effects, some reports reveal that these isoforms may modulate different signaling cascades in the liver and skeletal muscle tissues (Long et al. 2011; Eckstein et al. 2017). Also, studies using knockout mice suggest that IRS1 and IRS2 modulate different physiological processes (Shirakami et al. 2002; Dong et al. 2006; Guo et al. 2009; Long et al. 2011; Costello et al. 2012). However, these differences have not been fully established within the central nervous system, and some paradoxical results indicate that whereas brain insulin resistance is mediated by dysfunctional IRS1 (Yarchoan et al. 2014) in other systems the reduction of the IGF1/IRS2 pathway is suggestive of a neuroprotective role (Freude et al. 2009; Sadagurski et al. 2011). In optimal conditions, both IRSs are considered equally activated by the insulin and the IGF1 receptors tyrosine kinase activity. Once these scaffolding proteins are phosphorylated in Tyr residues, they activate downstream signaling cascades, ie the PI3K/Akt pathway, Ras/ERK; and soluble tyrosine kinases from the Fyn family (Rondinone et al. 1997; Yamada et al. 1997; Hanke and Mann 2009; Whelan et al. 2010; Costa et al. 2012; Hemmings and Restuccia 2012; Machado-Neto et al. 2018). Several physiopathological conditions induce resistance to insulin and IGF1. Among others, neuroinflammation feedforward inhibition induces IRS1 phosphorylation in serine residues by cytokines like IKK $\beta$ and JNK. In hyperinsulinemia, feed-back inhibition via mTOR also phosphorylates IRS1 in Ser residues (Pederson et al. 2001; Aguirre et al. 2002; Copps and White 2012). Interestingly, both chronic insulin and IGF1 resistance in the brain are associated with the occurrence of Alzheimer's disease (AD) (Moloney et al. 2010; Dineley et al. 2014). Furthermore, reduced responses to IR-IRS1 and IGF1R-IRS2 linked to PI3 kinase (Talbot et al. 2012) have been found in postmortem analysis in AD patients, compared to controls.

The specific and differential role of IRS1 or IRS2 isoforms in distinct brain networks, isolated from other comorbidities, such as chronic inflammation or AD linked genetic mutations, has not been fully established. Whether insulin resistance is a primary cause of cognitive dysfunction or it is a consequence of inflammatory conditions remains elusive. Thus, we aimed to understand the specific role of IRS1 in the adult healthy brain, in the absence of any other peripheral or central comorbidities. To that end, we made use of AAV expressing shRNA targeting IRS1 into the dorsal hippocampus of female and male rats. A rat model has been extensively used to study brain function with well-accepted methods of behavioral evaluation. We evaluated the IRS1 silencing effect in specific hippocampal circuitries involved in spatial memory and the synaptic plasticity of infected neurons. We report for the first time that, downregulation of IRS1 in dentate gyrus hilar Parvalbumin and Somatostatin neurons impair spatial but not social memory. In addition, our results suggest that female rats may be more susceptible to brain insulin resistance than males, which may contribute to explain sex differences in the prevalence of neurodegenerative diseases.

\section{Materials and methods}

\section{Design and generation of shRNA constructs.}

Several sequences specifically targeting IRS1 were designed using the provided software on the Invitrogen webpage (https://rnaidesigner.thermofisher.com/ rnaiexpress/); parameters were modified to fit 
destination plasmid for shRNA cloning (pSM155). Best shIRS1 candidates were synthesized (ThermoFisher Scientific $\left.{ }^{\circledR}\right)$. Two shRNA constructs were cloned following the protocol described (Du et al. 2006). The sequences of the oligonucleotides used in this study were as follows:

\section{Sh-IRS1A}

Top

5`TGCTGGCAGGCACCATCTCAACAATCGTTTTGGCCACTGACTGACGATTGTTGAGATGGT GCCTGC 3

Bottom

5'CCTGGCAGGCACCATCTCAACAATCGTCAGTCAGTGGCCAAAACGATTGTTGAGATGGT GCCTGCC 3'

Sh-IRS1B

Top

5’TGCTGGCCTGGAGTATTATGAGAACGGTTTTGGCCACTGACTGACCGTTCTCATAATACT CCAGGC 3'

Bottom

5'CCTGGCCTGGAGTATTATGAGAACGGTCAGTCAGTGGCCAAAACCGTTCTCATAATACTC CAGGCC3'

Construction and purification of recombinant AAV-CMV-shIRS1A-EGFP

In order to generate the expression cassette, a PCR (Supreme NZYProof DNA polymerase, NZYtech, Lisbon, Portugal) targeting the shIRS1 and the EGFP was performed. Primers were designed with the ClaI and HindIII restriction sites. The PCR product was cloned into pJET 1.2 according to the manufacturer's instructions (CloneJET PCR Cloning Kit; ThermoFisher Scientific ${ }^{\circledR}$, MA, USA). Insert was cloned by ClaI/HindIII enzyme restriction from the pJET 1.2 constructs and subcloned into the destination vector pAAV-CMV-GFP (Cell Biolabs INC, CA, USA) by ligation (T4 DNA Ligase; Takara Bio INC, Kyoto, Japan) overnight. Ligation was transformed into Endura ${ }^{T M}$ competent cells (Lucigen, WI, USA), and positive colonies evaluated by $M s c I$ and/or EcoRV enzyme digestion. Finally, sequencing confirmed the absence of point mutations (Servicio Central Soporte Investigacion Experimental (SCSIE) from the University of Valencia). pAAV-CMV-shIRSIA-EGFP was co-transfected with pHelper and pAAV2-Dj/8 (Cell Biolabs, INC.) into packaging HEK293 cells to produce recombinant AAV2/DJ8CMV-shIRS1A-EGFP. The resulting viral particle was purified by the iodixanol method as described in Addgene protocols for AAV production and purification. Cell lines were purchased from Cell Biolabs, $I N C$, and were free from mycoplasma.

Animals and surgical procedures

Female and male Wistar rats (Janvier Labs, Saint-Berthevin, France) between 250 and 550g were kept at the animal facility of the University Jaume I. The procedures followed the directive 86/609/EEC of the European Community on the protection of animals used for scientific purposes. The experiments were approved by the Ethics Committee of the University Jaume I. (scientific procedure 2019/VSC/PEA/0143). The animals were maintained on a $12 \mathrm{~h}$ light cycle and provided with food and water ad libitum. Animals were caged in pairs to reduce single caging -induced stress. Surgeries were done over one week, approx. 4 surgeries a day. Female and male cohorts were done in different phases to avoid confounding results. 
For surgical procedures rats were first deeply anesthetized with ketamine (Ketamidor $50 \mathrm{mg} / \mathrm{kg}$ i.p.; Merial Laboratories SA, Barcelona, Spain) and xylazine (Xilagesic 10 mg/kg i.p.; Lab Calier, Barcelona, Spain). Stereotaxic coordinates used to infuse the viral particle into the dorsal hippocampus bilaterally were AP $-4.36 \mathrm{~mm}, \pm \mathrm{ML} 2.5 \mathrm{~mm}$, DV $-2.5 \mathrm{~mm}$ from bregma (Paxinos and Watson 2013). Bilateral injections were performed; $2 \mu \mathrm{L}$ of either AAV/DJ8-CMV-EGFP $(4.94 \mathrm{E}+11 \mathrm{GC} / \mathrm{mL})$ or AAV/DJ8-CMVshIRS1-EGFP $(1.02 \mathrm{E}+11 \mathrm{GC} / \mathrm{mL})$ per hemisphere. Animals were randomly divided into AAV control AAV shrIRS1 groups and code labeled by an independent researcher. We used 7-8 rats per group and gender. This group size has been chosen based on our previous experience and the literature. Animal welfare was monitored although the procedure according to Ethical committee rules. Table and graph showing the subject's weight before the surgery and one month later, before behavior tests are shown in supplementary table S2.

\section{Behavioral procedures}

In all the procedures rats were habituated to the testing room two days before performing the behavior paradigm. Animals were recorded using a video tracking system (Smart 2.5.19, Panlab, Barcelona, Spain). Behavioral tests were conducted during the day, with a dim light. The researcher was blind to the group's condition. All animals performed these tests.

Open Field; Rats were placed in the open field facing one of the walls and allowed to freely explore the arena for $10 \mathrm{~min}$. The latency (time) to cross for the first time the center quadrants with all four legs was quantified.

Novel Object Recognition (NOR); NOR experiment was conducted as described previously (SánchezSarasúa et al. 2016). Briefly, rats were habituated to the experimental box before the test day. In the test day, the subjects were left to explore two identical objects, for 3 minutes (familiarization phase). After $1 \mathrm{~h}$ interval, the rats were back to the arene, and allowed to explore one of the previous objects (familiar) and a novel object for 3 min (test phase). "Exploration" is defined as time spent sniffing the object closer than $4 \mathrm{~cm}$, or touching it with the head oriented towards the object. Climbing over the object or running around it was not considered exploration. Data were expressed as the discrimination Index (DI), defined as the time spent in the novel object, minus time spent in familiar one, divided by total time exploring. Thus, DI $=0$ means the animal spends equal time exploring both objects. $\mathrm{DI}=1$ or $\mathrm{DI}=-1$ would mean that the subject spends total time exploring the novel or the familiar, respectively.

T-maze; The T-maze test was performed as described by Sánchez-Sarasúa et al. 2016. Briefly, the animal is placed in the starting position, and left to explore for $5 \mathrm{~min}$, with access to two of the three arms (familiarization phase). The rats were then returned to the home cage for a 2-h inter-trial interval and then placed back in the starting position, but now with access to all three arms for 5 min (test phase). The arm that was closed during the familiarization phase is considered the "novel" arm, the arm visited during the familiarization phase, is considered the "familiar" arm. Data expressed as the DI, as above.

Forced Swim Test; The forced swim test (FST) apparatus consisted of a Plexiglass cylinder with translucent walls $\left(30 \mathrm{~cm}\right.$ in diameter and $50 \mathrm{~cm}$ high). The cylinder was filled with tap water at $23 \pm 1{ }^{\circ} \mathrm{C}$ and the water depth was adjusted so the rat cannot touch the bottom of the container. In the first session (pre-test phase), the rats were placed in the water-filled cylinder for $15 \mathrm{~min}$. Twenty-four hours later (test phase), each rat was returned to the FST apparatus for 5 min (Yankelevitch-Yahav et al. 2015). Once each session was finished, the animals were placed in a drying cage with a heating pad under it and constant temperature that did not exceed $37^{\circ} \mathrm{C}$. Both days, the cylinder was placed at the same position in the room and the water was changed after every test to avoid any influence on the next rat. Three parameters were measured: "Immobile" was considered when the rat was floating without any movement, 
"Climbing" when the limbs were moved quickly breaking the surface of the water, "Swimming" when the rat moved the limbs in a paddling way.

\section{Immunofluorescence}

Immunofluorescence was performed as described previously (Ribes-Navarro et al. 2019). Briefly, randomly chosen rats were anesthetized and transcardially perfused with saline $(0.9 \% \mathrm{NaCl})$ followed by fixative (4\% paraformaldehyde in $0.1 \mathrm{M} \mathrm{PB}, \mathrm{pH} 7.4$ ). The other half of the brains were frozen for biochemical analysis. However, given the specificity of the AAV infection, considering that the surrounding neurons do express mRNA IRS1 we did not perform biochemical analysis.

After perfusion, the brains were removed, postfixed overnight and cryoprotected in $30 \%$ sucrose in 0.01 M PBS pH 7.4 for 3 days. The brains were cut in rostrocaudal direction $(40 \mu \mathrm{m})$ using a sliding microtome Leica SM2010R (Leica Microsystems, Heidelberg, Germany). The following primary antibodies were used: goat anti-ChAT (MERCK Millipore, Darmstadt Germany; 1:700); mouse anticalbindin (CB) (Swant, Marly, Switzerland; 1:3,000); rabbit anti-calretinin (CR) (Swant; 1:2,000); rabbit anti-parvalbumin (PV) (Swant; 1:5,000); mouse anti-somatostatin (SOM) (Santa Cruz Biotechnology, Santa Cruz, CA, USA; 1:50); mouse anti-synaptophysin (Sigma-Aldrich, St Louis, MO, USA; 1:100); mouse anti-Glial Fibrillary Acidic Protein (GFAP) (Sigma-Aldrich, St Louis, MO, USA; 1:100); rabbit anti-IRS1 (Novus Biologicals, Abingdon, UK; 1:100). After overnight incubation, AAV-infected sections were rinsed and incubated for $2 \mathrm{~h}$ at RT with donkey anti-mouse $\mathrm{Cy} 3$, donkey anti-goat $\mathrm{Cy} 3$, and goat anti-rabbit Cy3 secondary antibodies (Jackson Immunoresearch, Suffolk, UK). Finally, sections were mounted on slides and coverslipped using Fluoromount-G mounting medium (Invitrogen, California, USA). For descriptive immunofluorescence, three animals per condition were done, and one example is shown in results. For IRS1+EGFP dual-labeling quantifications female rats 3 controls and 4 shIRS1 and male rats 7 controls and 7 shIRS 1 were used and 2 dentate gyri per animal. For CR+AAV, PV+AAV, and SOM+AAV dual-labeling quantifications female rats 4 shIRS 1 and male rats 7 shIRS1 were used and 1 dentate gyrus per animal. For synaptophysin quantifications female rats 3 controls and 4 shIRS1 were used and 10 different axonal fibers per animal.

\section{Imaging and analysis}

Fluorescence images were taken with a confocal scan unit with a module TCS SP8 equipped with argon and helio-neon laser beams attached to a Leica DMi8 inverted microscope (Leica Microsystems). Excitation and emission wavelengths for $\mathrm{Cy} 3$ were 433 and 560-618 nm respectively; Alexa488-labeled excitation wavelength was $488 \mathrm{~nm}$ and its emission at $510-570 \mathrm{~nm}$. Serial $0.2 \mu \mathrm{m}$ scans were obtained in the Z-plane.

For the quantification of dual-labeling, we used a 20x lens. Image J software combined with loci. tools plugin (BIO-FORMATS, University of Wisconsin-Madison) was used to count the number of positively labeled cells in 20-32 Z-plane sections from control and shIRS1 groups of both females and males. For IRS1+EGFP dual-labeling quantifications, data were expressed as the percentage of double-labeled neurons (IRS1+AAV), divided by the total number of AAV-positive neurons. 3 AAV controls and 4 AAV shIRS1 females and 7 AAV controls and 7 shIRS1 males were analyzed by a researcher blind to the condition (2 dentate gyri per animal). For CR+EGFP, PV+EGFP, and SOM+EGFP dual-labeling quantifications, data were expressed as the percentage of double-labeled neurons (neuronal marker + EGFP), divided by the total number of neuronal marker-positive cells. 4 AAV shIRS1 females and 7 AAV shIRS1 males were analyzed by a researcher blind to the condition (1 dentate gyrus per animal).

For quantification of synaptophysin signal in AAV infected neurons, we used a $63 \mathrm{x}$ lens. Image $\mathrm{J}$ software combined with loci. tools plugin was used to count the number of synaptophysin points 
(minimum 3 pixels were considered) in one AAV-positive fiber in 10-15 Z-plane sections from control and shIRS1 groups. Three AAV controls and four AAV shIRS1 female animals, 10 different axons per animal were analyzed by a researcher blind to the condition. Data is calculated as the number of synaptophysin positive signals on the AAV-infected fiber, normalized to the area of the fiber.

The experimental outcomes from these procedures were, IRS1 mRNA expression, done in transfected cells. Behavioral changes in rats inoculated with the virus. And postmortem analysis of AAV tracing, colocalization quantification studies, and synaptophysin quantification.

\section{Statistical analysis}

The analysis was carried out with Graph Pad software (GraphPad Prism V8 software, GraphPad, La Jolla, CA, USA). Data reported as the mean \pm SEM and the " $n$ " the number of independent subjects. Data were subjected to Kolomogorov-Smirnov test for Gaussian distribution. If normality was confirmed, one-tailed or two-tailed Student's $t$-test with probability set at $\alpha<0.05$ was used, otherwise the non-parametric Mann-Withney T-test was applied as indicated. Pearson test was used for correlation analysis. The correlation coefficient $r$ values $( \pm 0.1$ to \pm 0.3 ) reveal weak, values around $( \pm 0.3$ to \pm 0.5$)$ reveal medium and higher than \pm 0.5 reveal a strong correlation. Positive or negative $r$ values mean positive or negative correlations, respectively.

\section{Results}

shRNA IRS1 knocks down IRS1 mRNA levels in HEK293 cells

To verify the knockdown efficiency of the shRNA against IRS1, we measured IRS1 and IRS2 mRNA expression in transfected HEK293. We used two constructs, expressing two different shRNA sequences (shIRS1A and shIRS1B) compared to plasmid control. We observed that expression of IRS1 but not IRS2 mRNA was significantly reduced by both sequences (Fig S1). The shRNAs were designed against rat, but they displayed $98 \%$ homology with humans, so we could translate the results from human cells to rat IRS1.

$A A V$-shIRS1-EGFP injection in dorsal hippocampus alters spatial memory, especially in females, but not social memory, nor anxiety or depressive symptoms

AAV-shIRS1 and AAV control (Fig 1A) were generated in HEK293 cells with a titration of 1.02E+11 $\mathrm{GC} / \mathrm{mL}$ and $4.94 \mathrm{E}+11 \mathrm{GC} / \mathrm{mL}$, respectively. AAV particles were bilaterally injected by stereotaxis in the dorsal hippocampus (AP -4.36 mm, \pm ML $2.5 \mathrm{~mm}$, DV $-2.5 \mathrm{~mm}$ from Bregma) (Fig 1B). Control and shIRS1 injected animals were subjected to behavioral paradigms one month after viral particle injection (Fig 1C). Both paradigms evaluate the memory in rodents exploiting their innate exploratory preference for novel stimuli, the NOR test examines the capability of the animal to remember a familiar object. To quantify it, the discrimination index (DI) is obtained by subtracting the time exploring the familiar object to the time exploring the novel one; divided by total exploring time. We observed that the shIRS1 injected females animals (DI; $0.24 \pm 0.13$ ) discriminate both objects significantly less than the AAV control injected animals (DI; $0.56 \pm 0.11$ ); whereas we did not see the same effect in males receiving shIRS1 (DI; $0.44 \pm 0.05$ ), compared to controls (DI; $0.47 \pm 0.06$ ) (Fig 2A). The data is represented by mean \pm SEM and analyzed using unpaired Student's t-test $(* \mathrm{p}<0.05)$.

In the T-maze test, we recorded the number of entries and the time exploring the novel arm. Females in the shIRS1 group showed reduced DI $(-0.03 \pm 0.10)$ in the time spent in novel arms compared to controls DI $(0.23 \pm 0.08)$ Fig 2B. Similarly, in males, shIR1 injected male rats had significantly lower DI value ($0.00 \pm 0.04)$ than controls $(\mathrm{DI} ; 0.16 \pm 0.07)$ in the time spent in the novel arm. Females showed 
significantly lower DI $(0.02 \pm 0.02)$ in the number of entries (Fig $2 \mathrm{C})$ to the novel arms compared to the control group DI $(0.14 \pm 0.06)$. Also, in males, the number of entries to the novel arm was lower (DI; $0.02 \pm 0.03$ ) than controls (DI; $0.09 \pm 0.04$ ). These results suggested that the downregulation of IRS1 impairs the memory of the familiar arm, both in female and male rats. We evaluated anxiety by the latency to cross for the first time the open filed and observed lower but not significantly different shIRS1 (43.13 \pm 11.06$)$ compared to control AAV $(61.67 \pm 22.48)$ injected females. Nor differences either in

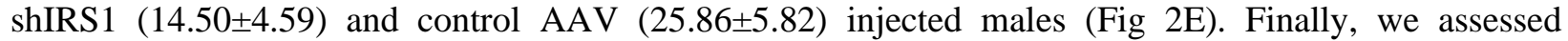
depressive symptoms in the animals by the forced swimming test. As above, hippocampal injection of

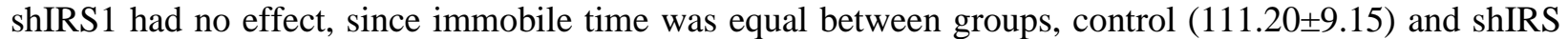

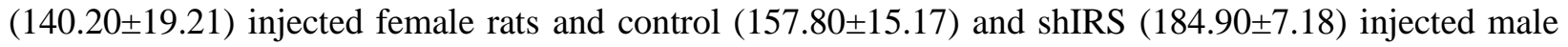
rats (Fig 2F). Swimming time and climbing were not different either between groups (data not shown).

\section{AAV DJ8 track dorsal hippocampus efferent connections}

Dorsal hippocampus efferent projections reach Medial Septum (MS) and mammillary bodies through the fimbria-fornix white track (Fig 3A). We inoculated AAV-CMV-shIRS1-EGFP and AAV-CMV-EGFP in the dorsal hippocampus. We observed fibers and cells in the dorsal subiculum (DS), an area within the hippocampal formation (Fig 3B), and in the polymorphic area or hilus of the dentate gyrus (PoGD) (Fig 3C). As expected, we found fibers in the fornix (Fig 3D) and consequently in MS (Fig 3E) and mammillary bodies (Fig 3F).

\section{AAV-shIRS1-EGFP labels dorsal specific hippocampal neurons}

AAV infected mostly neurons in the hilus of the dentate gyrus (DG), but not the granule layer (Fig 4). To a lesser extent, the AAV entered the CA1 (Fig S2). We observed that astrocytes were not infected by the AAV tropism (Fig S3). Diverse GABAergic cell types localize in the DG, so we performed immunostaining of neuronal markers to characterized AAV infected neurons. We found that AAV labels co-localized with calretinin (CR) (Fig 4B), parvalbumin (PV) (Fig 4C), and somatostatin (SOM) (Fig 4D) neurons. The quantification of the EGFP-cell marker double labeling revealed that $48.78 \% \pm 2.97$ of SOM cells and $42.88 \% \pm 3.75$ of PV had been infected with AAV, whereas the number of infected CR cells was slightly lower; $32.30 \% \pm 3.39$, suggesting that the SOM and PV neuronal activity has been more affected by IRS1 silencing (Fig 4E).

\section{$A A V$ axons in the medial septum reach $P V$ and ChAT occupied areas}

We observed that MS was strongly innervated by AAV-labeled axons. The fibers seem to be restricted to the most medial part. To evaluate the type of neurons potentially regulated by the AAV labeled projections, we carried out a series of immunolabeling analysis. We observed that AAV projections colocalized mostly within PV (Fig 5B) and cholinergic (ChAT) (Fig 5C) area. Also, sparse calbindin (CB) positive neurons fall within the axon labeled areas (Fig 5D). The field where calretinin (CR) neurons appear is completed devoid of EGFP fibers (Fig 5E).

$A A V$-shIRS1 results in lower expression of IRS1 in the dorsal hippocampus and correlates with behavior.

To ascertain if AAV-shIRS1 corresponded to reduced IRS1 protein expression, double IRS1 immunofluorescence and EGFP expression were quantified and expressed in relation to total EGPF positive neurons (Fig 6B). We observed that the AAV-shIRS1 resulted in a reduced number of double IRS1-EGFP neurons $(67.88 \% \pm 1.22)$, compared to control AAV $(80.28 \% \pm 1.9)$. This result is indicative of a knockdown of IRS1 expression in the dentate gyrus were the AAV-shIRS1 was inoculated. 
Moreover, to elucidate if the different degrees of reduced expression could account for the behavioral variability, we performed linear regression and applied the Pearson test, correlating the DI in time spent in the novel arms (T-maze) with the degree of IRS1 reduction (as shown in Fig 6B). We observed that the correlation between DI time in novel arms in the T-maze, was positive (Correlation DI time with shIRS1 $\mathrm{r}=0.63$, and DI time vs control $(\mathrm{r}=-0.15)$ (Fig S4). These results suggest that in AAV-shIRS1 subjects the degree of IRS1 reduction correlates better with the behavioral impairment than in control subjects.

\section{$A A V$ ShIRS1 infection results in lower synaptophysin positive labels compared to AAV control}

To explain the possible mechanism underlying the observed behavioral results we hypothesized that the reduction in IRS1 signaling could impair synaptic plasticity, based on previous data where insulin resistance reduced synaptophysin expression (Neves et al. 2018) and reduced plasticity (Spinelli et al. 2017, 2019). To evaluate the effect of shIRS1 in axons in the MS area (Fig 7A), we quantified synaptophysin labeling normalized by the green-labeled area. We observed that the number of synaptophysin positive labels in axons was significantly lower in AAV-CMV-shIRS1-EGFP axons

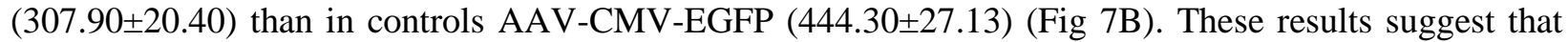
knocking down IRS1 reduces synaptic plasticity, as measured by synaptic vesicle proteins, used as an indirect marker of synaptic activity. Representative images are shown for AAV control (Fig 7C) and AAV-shIRS1 (Fig 7D)

\section{Discussion}

In our study, we show that infection of shIRS1 using an AAV2/DJ8 derived virus inoculated in the hippocampus area affects hippocampal memory formation in female and male rats. It does not alter anxiety parameters in any gender. Loss of spatial memory is one of the first symptoms in Alzheimer's disease (AD), caused by progressive loss of neuronal circuitries. Diabetes, dyslipidemia, and cardiovascular factors have been demonstrated to play a role in degenerative diseases like AD and frontotemporal dementias (Golimstok et al. 2014). These pathologies are linked to brain insulin resistance, which triggers cognitive decline and Alzheimer's (Folch et al. 2019). Most research addressing insulin resistance in the brain assumes that neuroinflammation causes insulin resistance, and this, in turn, aggravates neuronal damage, stimulating the neuroinflammatory process. Although neuroinflammation is a general process affecting the whole brain, in $\mathrm{AD}$ patients one of the first clinical symptoms is the loss of spatial memory. Thus, it is likely that only particular circuitries involved in this memory are affected at the early stages of the disease before massive neuronal death occurs. Major hippocampal circuitries like the septo-hippocampal connections (Khakpai et al. 2013) and the circuit of Papez (Aggleton et al. 2016) are crucial for hippocampal-dependent memory formation.

In this study, we made use of an AAV carrying an shRNA targeting IRS1. We confirmed mRNA downregulation by PCR in vitro studies and reduced protein expression in vivo by immunofluorescence. We also confirmed that the shIRS1 did not affect significantly IRS2 expression, so the knocking down is specific for this isoform. Most studies in the literature have focused on the IRS1 inactivation due to serine phosphorylation. Serine residues are phosphorylated by inflammatory signals. In this study we have used shIRS1 to reduce the expression of IRS1, thus IRS1 signaling activity is reduced. This study is not a knockout, so a residual activity is present, and there are no compensations either which may alter the specific effect of an IRS1 reduced expression. Importantly, we wanted to isolate the IRS1 effect, since inflammatory processes affect several pathways, one of the IRS1, we aimed to examine the IRS1 contribution in discrete neuronal pathways in the absence of neuroinflammation. This may indicate a way to improve brain health in human pathologies where IRS1 signaling is affected.

Females rats inoculated with the AAV-CMV-shIRS1-EGFP performed significantly worse in the novel object recognition test (NOR), compared to controls inoculated with AAV-CMV-EGFP. The NOR test 
evaluates the subject memory by measuring the recognition of familiar objects. When the animal does not remember having explored an object, it will explore both equally, as they are both equally new to the subject. Unlike female subjects, males inoculated with shIRS1 remember the familiar object, and they behaved similarly to control injected males. This result suggests that the female brain may display a higher susceptibility to insulin resistance, this would agree with the observation that AD is more prevalent amongst females (Cao et al. 2019). In humans, AD is reported to be more prevalent in women than in men (Yan et al. 2018). Reports indicate that around 70\% of AD patients are women in the United States (Hebert et al. 2001; Miyawaki 2015) and in Europe (Beam et al. 2018). Several hypotheses have been proposed to explain that fact. The most evident is that women have a longer life span than men, but even after normalizing to it, other biological factors have been considered. Amongst others, female higher sensitivity to the presence of one ApoE4 allele has been proposed (Payami et al. 1994; Ungar et al. 2014), since this isoform increases the probability to suffer from $\mathrm{AD}$ (Uddin et al. 2019). ApoE is the major lipid transporting molecule in the brain and the isoform ApoE4 has less capacity to shuttle lipids than the others, thus, given the importance of cholesterol in neuronal function (Marcuzzi et al. 2018), cholesterol dysregulation is expected to contribute to degenerative pathologies (Jeong et al. 2019). Because dyslipidemia and insulin resistance act in a vicious cycle to promote pathologic processes contributing to degenerative diseases; under the light of our results, we proposed that females, even with normal estrogen levels (in this study female rats were not ovariectomized) may be more susceptible to insulin resistance (due to reduced IRS1 function) than male. We hypothesize that this could be a major contributing factor to the higher prevalence of $\mathrm{AD}$ in women, however, further studies are required to unveil the underlying mechanism to this effect.

The T-maze evaluates the spatial navigation capability. Exploration of novel spaces is a hallmark of rodent, and as expected, female and male subjects inoculated with control AAV explored the new arm longer time than the familiar one. Both females and males injected with shIRS1 failed to discriminate between the maze novel and familiar arms, indicating that they do not remember the arm previously visited. These results agree with former studies reporting that insulin receptor silencing using a lentiviral derived particle inoculated into the hippocampus impaired spatial memory (Grillo et al. 2015). Importantly, in this task, we found no difference between male and female subjects. One possible explanation for this result is that the T-maze can be considered a more difficult task than the NOR task, it also measures navigation and NOR evaluates recognition. Therefore, we hypothesize that the NOR test may more sensitive to smaller neurological deficiencies (Sánchez-Sarasúa et al. 2016).

We observed no differences in anxiety-like behaviors. This may be in contrast with previous reports indicating that hippocampus-septal connections may regulate anxiety (Degroot and Treit 2002, 2003). Because the circuit targeted with the AAV DJ8 virus has been proved very specific, we consider that we have only affected the hippocampal processing of spatial memories.

In this study, AAV DJ8 inoculated in the hippocampal area is mostly found in neurons within the Polymorphic area (PoGD) or hilum of the dentate gyrus (DG) and to a lesser extent in the dorsal subiculum (DS). Sparse neurons were found in the CA1 regions (Fig S3). We observed abundant fibers via the fimbria-fornix pathway. This pathway is a white track that contains hippocampal efferents (to MS and mammillary bodies) and afferents (from septal cholinergic projections (Gage and Björklund 1986)). Consistent with hippocampal efferent we observed abundant fibers through the fornix. Following Papez circuitry we also found fibers in the mamillary bodies. Consistently, no thalamic staining was observed (data not shown). In addition, we found extensive green fibers in the medial septum (MS). Traditionally, MS has been considered to receive projections from CA1 GABAergic somatostatin (SOM) neurons (Ujfalussy et al. 2007). However, long-range projection interneurons have been localized in the DG hilum innervating the MS (Jinno 2009). Recently, these DG long-range projection interneurons have been characterized as a new type of SOM interneuron and named as hilar interneurons with long axons (HIL). HIL neurons co-exist with hilar SOM interneurons (the SOM HIPP neurons) (Yuan et al. 2017). Long 
axon SOM neurons provide regulation to both nearby Parvalbumin (PV) interneurons and display collaterals projecting to the MS, where they contact ChAT and PV neurons (Yuan et al. 2017).

We cannot rule out the effect of IRS1 silencing in glutamatergic neurons in the hippocampus. However, since we have seen a high proportion of AVV infection in the hilum of the dentate gyrus, where most neurons are GABAergic, for clarity, we decided to focus this study on the GABAergic connections. This rationale is backed up by the strong green labeling we observed in the PV area within the MS, which corresponds to hippocampal SOM neurons projecting to the septal area. This, by no means, suggests that it is the only mechanism underlying the IRS role in neuronal activity. To better understand the role of IRS1 in Glutamatergic, different AAV serotypes may improve infection in the glutamatergic type of neurons.

We immunocharacterized the neurons within the DG infected with AAVDJ8 and confirmed the colabeling with the SOM antibody. Moreover, AAV fibers were found where the PV and ChAT neurons are in the MS (Yuan et al. 2017), therefore, we assume that our AAV can be affecting both types of hilar SOM, the short and long projecting neurons. Both types of hilar SOM interneurons play an important role in regulating hippocampal function. The DG is the first phase of the classical tri-synaptic circuit (Eichenbaum 1993; Milenkovic et al. 2013b), defined as the circuit starting from the entorhinal cortex to the CA3 pyramidal neurons, and from these to the CA1. This circuit is crucial for the formation of new memories (Burgess et al. 2002; Leutgeb et al. 2005). Thus, information coming from the entorhinal is regulated with precise timing by the strong inhibitions within the DG, this modulates the output of granule cells to CA3, where spatial memory forms a spatial pattern. HIPP provides dendritic inhibition controlling the entrance of spatial information from the entorhinal cortex to the DG. In addition, the inhibition mediated by HIL cells is proposed to contribute to the temporal coordination of MS and local rhythmic DG activity (Yuan et al. 2017). Since we do not see soma labeling in the MS, we assume that AAV only travels anterogradely through the fimbria-fornix to MS, therefore, we assume that MS neuronal activity is affected via hilar SOM long projections.

We found that axons from the hippocampus innervating MS (the long projecting SOM) have reduced synaptophysin labeling, indicative of reduced synaptic plasticity. Even though we cannot rule out other mechanisms, this result illustrates that the reduction in IRS1 function impairs long projecting SOM neuronal plasticity with behavioral consequences. Synaptophysin labeling is a marker of presynaptic activity, together with postsynaptic markers it is considered a measure of the circuit synaptic plasticity. Presynaptic alterations of synaptophysin labeling have been reported as an early event in Alzheimer's disease (Yuki et al. 2014).

We speculated that IRS1 silencing may alter neuronal presynaptic activity, and even if we cannot rule out an alteration at the postsynaptic level, we focused on the connection hippocampus-septum, and we used synaptophysin labeling as a presynaptic marker of synaptic activity. Indeed, we found that synaptophysin staining was reduced in shIRS1 infected neurons compared to controls. The mechanism underlying this observation can be a reduced IRS1-PI3-AKT pathway, that regulates Glut 4 translocation to the membrane (Grillo et al. 2009), which would hamper glucose availability at the nerve terminals, thus impairing synaptic activity (Ashrafi et al. 2017). The long-term modulation of synaptic activity is achieved (amongst others) by regulating the expression of several neurotransmitter vesicle proteins. This has been shown with a high-fat-diet model that reduced IGF1 and synaptophysin levels. These were recovered after IGF signaling activation (Li et al. 2019). Also, brain-derived neurotrophic factor (BDNF) has been shown to modulate synaptic plasticity by regulating synaptophysin expression (Tartaglia et al. 2001; Zhang et al. 2017).

IRS1 has been mostly studied in the context of insulin and IGF1 signaling, however, given their scaffolding function it is plausible that IRS mediate several neurotrophic factors, including BDNF 
(Yamada et al. 1997). This would agree with the fact that even a small reduction in the IRS1 expression function has resulted in a behavioral defect.

In addition to SOM, we found co-labeling with GABAergic PV interneurons, and to a lesser extent with CR neurons. Fast spiking PV interneurons regulate network oscillation and synchrony (Milenkovic et al. 2013a). Previous reports demonstrate that PV dysregulation in the DG affects memory and anxiety (Zou et al. 2016). However, in our present work, we do not see alterations in anxiety nor social behavior or depressive symptoms, but specifically spatial memory.

Under the light of our results, we hypothesized that IRS1 signaling is required for both SOM and PV inhibitory action, by reducing synaptic plasticity. We believe that we have affected two pathways by the AAV infection; on one hand, there may be disinhibition of the information coming from the entorhinal cortex, hampering the formation of a clear spatial pattern in CA1. On the other hand, there may be disinhibition of PV and ChAT neurons in the MS, which in turn may increase PV and cholinergic action back into the hippocampus, this dysregulation can thus impair the excitatory/inhibitory balance.

Moreover, the Papez circuitry is essential to memory formation (Escobar et al. 2019). Papez circuitry starts in the subiculum, and it is part of the hippocampal formation. Evidence supports that the sequential pathway from the subiculum to the mammillary bodies via the fornix, and from there to the anterior thalamus, is required for spatial memory in rats (Gaffan et al. 2001) and humans (Gaffan and Gaffan 1991). Moreover, this circuitry is found severely affected in AD patients (Zarei et al. 2010). Because viruses do not "jump" to adjacent neurons, the effect of the IRS1 silencing is confined to the hippocampal projection to the mamillary bodies via the fornix. Some reports that have specifically disrupted this efferent projection indicate that it has only a mild effect on memory formation (Vann et al. 2011). Other studies, while emphasizing mammillary bodies function in memory formation (Vann and Nelson 2015), indicate that other projections from brain stem nucleus (eg the tegmental nuclei of Gudden), and not the hippocampal formation, are critical for sustaining mammillary body function (Vann 2010; Vann and Nelson 2015). Therefore, taking together these findings, we may assume that the effect we have observed disrupting memory formation in rats inoculated with AAV shIRS1 is most likely due to IRS1 knockdown in hilar DG GABergic neurons, modulating the incoming flow of information from the entorhinal cortex and projections to the MS.

Taking together all these findings, our works supports that IRS1 signaling in GABAergic hippocampal neurons, both interneurons, and MS projecting neurons is required for forming spatial memories. In this model, we mimic insulin resistance by silencing IRS1 in an otherwise healthy brain (i.e no inflammatory ambiance). In neurodegenerative diseases, inflammatory conditions induce insulin resistance, and concomitantly, loss of cognitive function. Our results indicate that insulin resistance, in the absence of inflammatory conditions, is sufficient to cause memory impairments, suggesting that this could be at the very onset of neurodegenerative diseases.

\section{Compliance with Ethical Standards}

The authors declare that the manuscript has not been submitted elsewhere.

Authors report no conflict of interest

\section{Funding statements.}

This work has been supported by Plan Propi from Universitat Jaume I UJI-B2018-01 and Conselleria de Educació, Cultura I sport AICO/2015/042 to AMSP. Predoctoral fellowship from Conselleria de Innovación, Universidades, Ciencia y Sociedad Digital ACIF/2016/250 to SSS. 
Ethical approval for animal experimentation.

The procedures followed the directive 86/609/EEC of the European Community on the protection of animals used for scientific purposes. The experiments were approved by the Ethics Committee of the University Jaume I (scientific procedure 2019/VSC/PEA/0143).

\section{Authors contribution.}

SSS performed the surgeries, conducted the behavioral paradigms, immunofluorescence, and image analysis, and contributed to manuscript writing; ARN generated the shRNA and AAV particles, did the PCR analysis, and contribute to writing of the manuscript; MTB contributed to the immunofluorescence studies; AMSP designed the study and wrote the manuscript.

\section{Acknowledgments.}

The authors want to thank all the people that generously donated to Crowdfunding Precipita (FECYT) that sponsored ARN, and the economic support from the Association of Alzheimer Families, AFA, Castellon.

\section{Informed consent.}

Not applicable

\section{References}

Aggleton JP, Pralus A, Nelson AJD, Hornberger M (2016) Thalamic pathology and memory loss in early Alzheimer's disease: moving the focus from the medial temporal lobe to Papez circuit. Brain 139:18771890. https://doi.org/10.1093/brain/aww083

Aguirre V, Werner ED, Giraud J, et al (2002) Phosphorylation of Ser307 in insulin receptor substrate-1 blocks interactions with the insulin receptor and inhibits insulin action. J Biol Chem 277:1531-1537. https://doi.org/10.1074/jbc.M101521200

Al-Delaimy WK, von Muhlen D, Barrett-Connor E (2009) Insulinlike growth factor-1, insulin-like growth factor binding protein-1, and cognitive function in older men and women. $\mathrm{J}$ Am Geriatr Soc 57:1441-6. https://doi.org/10.1111/j.1532-5415.2009.02343.x

Ashrafi G, Wu Z, Farrell RJ, Ryan TA (2017) GLUT4 Mobilization Supports Energetic Demands of Active Synapses. Neuron 93:606-615.e3. https://doi.org/10.1016/j.neuron.2016.12.020

Beam CR, Kaneshiro C, Jang JY, et al (2018) Differences between Women and Men in Incidence Rates of Dementia and Alzheimer's Disease. J Alzheimer's Dis 64:1077-1083. https://doi.org/10.3233/JAD180141

Bedse G, Di Domenico F, Serviddio G, Cassano T (2015) Aberrant insulin signaling in Alzheimer's disease: Current knowledge. Front Neurosci 9:1-13. https://doi.org/10.3389/fnins.2015.00204 
Burgess N, Maguire EA, O'Keefe J (2002) The human hippocampus and spatial and episodic memory. Neuron 35:625-641

Cao Q, Tan C-C, Xu W, et al (2019) The Prevalence of Dementia: A Systematic Review and MetaAnalysis. J Alzheimer's Dis 73:1-10. https://doi.org/10.3233/jad-191092

Cholerton B, Baker LD, Craft S (2013) Insulin, cognition, and dementia. Eur J Pharmacol 719:170-179. https://doi.org/10.1016/j.ejphar.2013.08.008

Copps KD, White MF (2012) Regulation of insulin sensitivity by serine/threonine phosphorylation of insulin receptor substrate proteins IRS1 and IRS2. Diabetologia 55:2565-2582. https://doi.org/10.1007/s00125-012-2644-8

Costa MM, Violato NM, Taboga SR, et al (2012) Reduction of insulin signalling pathway IRS-1/IRS2/AKT/mTOR and decrease of epithelial cell proliferation in the prostate of glucocorticoid-treated rats. Int J Exp Pathol 93:188-95. https://doi.org/10.1111/j.1365-2613.2012.00817.x

Costello D a., Claret M, Al-Qassab H, et al (2012) Brain deletion of insulin receptor substrate 2 disrupts hippocampal synaptic plasticity and metaplasticity. PLoS One 7:30-34. https://doi.org/10.1371/journal.pone.0031124

Degroot A, Treit D (2003) Septal gabaergic and hippocampal cholinergic systems interact in the modulation of anxiety. Neuroscience 117:493-501. https://doi.org/10.1016/S0306-4522 (02)00651-6

Degroot A, Treit D (2002) Dorsal and ventral hippocampal cholinergic systems modulate anxiety in the plus-maze and shock-probe tests. Brain Res 949:60-70. https://doi.org/10.1016/S0006-8993 (02)02965-7

Dineley KT, Jahrling JB, Denner L (2014) Insulin resistance in Alzheimer's disease. Neurobiol Dis $72 \mathrm{Pt}$ A:92-103. https://doi.org/10.1016/j.nbd.2014.09.001

Dong X, Park S, Lin X, et al (2006) Irs1 and Irs2 signaling is essential for hepatic glucose homeostasis and systemic growth. J Clin Invest 116:101-14. https://doi.org/10.1172/JCI25735

Du G, Yonekubo J, Zeng Y, et al (2006) Design of expression vectors for RNA interference based on miRNAs and RNA splicing. FEBS J 273:5421-5427. https://doi.org/10.1111/j.1742-4658.2006.05534.X

Eckstein SS, Weigert C, Lehmann R (2017) Divergent Roles of IRS (Insulin Receptor Substrate) 1 and 2 in Liver and Skeletal Muscle. Curr Med Chem 24:1827-1852. https://doi.org/10.2174/0929867324666170426142826

Eichenbaum H (1993) Thinking about brain cell assemblies. Science (80-. ). 261:993-994

Escobar I, Xu J, Jackson CW, Perez-Pinzon MA (2019) Altered Neural Networks in the Papez Circuit: Implications for Cognitive Dysfunction after Cerebral Ischemia. J Alzheimers Dis 67:425-446. https://doi.org/10.3233/JAD-180875

Folch J, Olloquequi J, Ettcheto M, et al (2019) The Involvement of Peripheral and Brain Insulin Resistance in Late-Onset Alzheimer's Dementia. Front. Aging Neurosci. 11 
Freude S, Hettich MM, Schumann C, et al (2009) Neuronal IGF-1 resistance reduces A $\beta$ accumulation and protects against premature death in a model of Alzheimer's disease. FASEB J 23:3315-3324. https://doi.org/10.1096/fj.09-132043

Gaffan D, Gaffan EA (1991) Amnesia in man following transection of the fornix: A review. Brain 114:2611-2618. https://doi.org/10.1093/brain/114.6.2611

Gaffan EA, Bannerman DM, Warburton EC, Aggleton JP (2001) Rats' processing of visual scenes: effects of lesions to fornix, anterior thalamus, mamillary nuclei or the retrohippocampal region. Behav Brain Res 121:103-17. https://doi.org/10.1016/s0166-4328 (00)00389-2

Gage FH, Björklund A (1986) Cholinergic septal grafts into the hippocampal formation improve spatial learning and memory in aged rats by an atropine-sensitive mechanism. J Neurosci 6:2837-47

Golimstok A, Cámpora N, Rojas JI, et al (2014) Cardiovascular risk factors and frontotemporal dementia: A case-control study. Transl Neurodegener 3. https://doi.org/10.1186/2047-9158-3-13

Grillo CA, Piroli GG, Hendry RM, Reagan LP (2009) Insulin-stimulated translocation of GLUT4 to the plasma membrane in rat hippocampus is PI3-kinase dependent. Brain Res 1296:35-45. https://doi.org/10.1016/j.brainres.2009.08.005

Grillo CA, Piroli GG, Lawrence RC, et al (2015) Hippocampal Insulin Resistance Impairs Spatial Learning and Synaptic Plasticity. Diabetes 64:3927-36. https://doi.org/10.2337/db15-0596

Guo S, Copps KD, Dong X, et al (2009) The Irs1 branch of the insulin signaling cascade plays a dominant role in hepatic nutrient homeostasis. Mol Cell Biol 29:5070-83. https://doi.org/10.1128/MCB.00138-09

Hanke S, Mann M (2009) The phosphotyrosine interactome of the insulin receptor family and its substrates IRS-1 and IRS-2. Mol Cell Proteomics 8:519-34. https://doi.org/10.1074/mcp.M800407MCP200

Hebert LE, Scherr PA, McCann JJ, et al (2001) Is the risk of developing Alzheimer's disease greater for women than for men? Am J Epidemiol 153:132-136. https://doi.org/10.1093/aje/153.2.132

Hemmings BA, Restuccia DF (2012) PI3K-PKB/Akt pathway. Cold Spring Harb Perspect Biol 4:a011189. https://doi.org/10.1101/cshperspect.a011189

Jeong W, Lee H, Cho S, Seo J (2019) ApoE4-Induced Cholesterol Dysregulation and Its Brain Cell TypeSpecific Implications in the Pathogenesis of Alzheimer's Disease. Mol Cells. https://doi.org/10.14348/molcells.2019.0200

Jinno S (2009) Structural organization of long-range GABAergic projection system of the hippocampus. Front Neuroanat 3:13. https://doi.org/10.3389/neuro.05.013.2009

Khakpai F, Nasehi M, Haeri-Rohani A, et al (2013) Septo-hippocampo-septal loop and memory formation. Basic Clin Neurosci 4:5-23

Kleinridders A, Ferris HA, Cai W, Kahn CR (2014) Insulin Action in Brain Regulates Systemic Metabolism and Brain Function. Diabetes 63:2232-2243. https://doi.org/10.2337/db14-0568 
Leutgeb S, Leutgeb JK, Barnes CA, et al (2005) Neuroscience: Independent codes for spatial and episodic memory in hippocampal neuronal ensembles. Science 309:619-623. https://doi.org/10.1126/ science. 1114037

Long YC, Cheng Z, Copps KD, White MF (2011) Insulin receptor substrates Irs1 and Irs2 coordinate skeletal muscle growth and metabolism via the Akt and AMPK pathways. Mol Cell Biol 31:430-41. https://doi.org/10.1128/MCB.00983-10

Machado-Neto JA, Fenerich BA, Alves APNR, et al (2018) Insulin Substrate Receptor (IRS) proteins in normal and malignant hematopoiesis. Clinics 73. https://doi.org/10.6061/CLINICS/2018/E566S

Marcuzzi A, Loganes C, Valencic E, et al (2018) Neuronal dysfunction associated with cholesterol deregulation. Int J Mol Sci 19. https://doi.org/10.3390/ijms19051523

Milenkovic I, Vasiljevic M, Maurer D, et al (2013a) The parvalbumin-positive interneurons in the mouse dentate gyrus express GABAA receptor subunits alpha1, beta2, and delta along their extrasynaptic cell membrane. Neuroscience 254:80-96. https://doi.org/10.1016/j.neuroscience.2013.09.019

Milenkovic I, Vasiljevic M, Maurer D, et al (2013b) The parvalbumin-positive interneurons in the mouse dentate gyrus express GABAA receptor subunits $\alpha 1, \beta 2$, and $\delta$ along their extrasynaptic cell membrane. Neuroscience 254:80-96. https://doi.org/10.1016/j.neuroscience.2013.09.019

Miyawaki CE (2015) Association of social isolation and health across different racial and ethnic groups of older Americans. Ageing Soc 35:2201-2228. https://doi.org/10.1017/S0144686X14000890

Moloney AM, Griffin RJ, Timmons S, et al (2010) Defects in IGF-1 receptor, insulin receptor and IRS$1 / 2$ in Alzheimer's disease indicate possible resistance to IGF-1 and insulin signalling. Neurobiol Aging 31:224-243. https://doi.org/10.1016/j.neurobiolaging.2008.04.002

Neves FS, Marques PT, Barros-Aragão F, et al (2018) Brain-defective insulin signaling is associated to late cognitive impairment in post-septic mice. Mol Neurobiol 55:435-444. https://doi.org/10.1007/s12035-016-0307-3

Paxinos G, Watson C (2013) The Rat Brain in Stereotaxic Coordinates: Hard Cover Edition. Elsevier Science

Payami H, Montee KR, Kaye JA, et al (1994) Alzheimer's disease, apolipoprotein E4, and gender. JAMA 271:1316-7

Pederson TM, Kramer DL, Rondinone CM (2001) Serine/threonine phosphorylation of IRS-1 triggers its degradation: possible regulation by tyrosine phosphorylation. Diabetes Jan;50(1):24-31. doi:

10.2337/diabetes.50.1.24

Ribes-Navarro A, Atef M, Sánchez-Sarasúa S, et al (2019) Abscisic Acid Supplementation Rescues High Fat Diet-Induced Alterations in Hippocampal Inflammation and IRSs Expression.

Mol Neurobiol. Jan;56(1):454-464. doi: 10.1007/s12035-018-1091-z.

Rondinone CM, Wang LM, Lonnroth P, et al (1997) Insulin receptor substrate (IRS) 1 is reduced and IRS-2 is the main docking protein for phosphatidylinositol 3-kinase in adipocytes from subjects with noninsulin-dependent diabetes mellitus. Proc Natl Acad Sci U S A 94:4171-5. https://doi.org/ 10.1073/ pnas.94.8.4171 
Sadagurski M, Cheng Z, Rozzo A, et al (2011) IRS2 increases mitochondrial dysfunction and oxidative stress in a mouse model of Huntington disease. J Clin Invest 121:4070-4081. https://doi.org/10.1172/JCI46305

Sánchez-Sarasúa S, Moustafa S, García-Avilés Á, et al (2016) The effect of abscisic acid chronic treatment on neuroinflammatory markers and memory in a rat model of high-fat diet induced neuroinflammation. Nutr Metab (Lond) 13:73. https://doi.org/10.1186/s12986-016-0137-3

Shirakami A, Toyonaga T, Tsuruzoe K, et al (2002) Heterozygous knockout of the IRS-1 gene in mice enhances obesity-linked insulin resistance: a possible model for the development of type 2 diabetes. $\mathrm{J}$ Endocrinol Aug;174(2):309-19. doi: 10.1677/joe.0.1740309.

Spinelli M, Fusco S, Grassi C (2019) Brain insulin resistance and hippocampal plasticity: Mechanisms and biomarkers of cognitive decline. Front Neurosci. Jul 31;13:788. doi: 10.3389/fnins.2019.00788.

Spinelli M, Fusco S, Mainardi M, et al (2017) Brain insulin resistance impairs hippocampal synaptic plasticity and memory by increasing GluA1 palmitoylation through. Nat Commun. Dec 8;8(1):2009. https://doi.org/10.1038/s41467-017-02221-9

Talbot K, Wang H-Y, Kazi H, et al (2012) Demonstrated brain insulin resistance in Alzheimer's disease patients is associated with IGF-1 resistance, IRS-1 dysregulation, and cognitive decline. J Clin Invest 122:1316-38. https://doi.org/10.1172/JCI59903

Uddin MS, Kabir MT, Al Mamun A, et al (2019). APOE and Alzheimer's Disease: Evidence Mounts that Targeting APOE4 may Combat Alzheimer's Pathogenesis. Mol Neurobiol 56:2450-2465. https://doi.org/10.1007/s12035-018-1237-z

Ujfalussy B, Kiss T, Orbán G, et al (2007) Pharmacological and computational analysis of alpha-subunit preferential GABA (A) positive allosteric modulators on the rat septo-hippocampal activity. Neuropharmacology 52:733-43. https://doi.org/10.1016/j.neuropharm.2006.09.022

Ungar L, Altmann A, Greicius MD (2014) Apolipoprotein E, gender, and Alzheimer's disease: an overlooked, but potent and promising interaction. Brain Imaging Behav 8:262-73. https://doi.org/10.1007/s11682-013-9272-x

Vann SD (2010) Re-evaluating the role of the mammillary bodies in memory. Neuropsychologia 48:2316-27. https://doi.org/10.1016/j.neuropsychologia.2009.10.019

Vann SD, Erichsen JT, O'Mara SM, Aggleton JP (2011) Selective disconnection of the hippocampal formation projections to the mammillary bodies produces only mild deficits on spatial memory tasks: implications for fornix function. Hippocampus 21:945-57. https://doi.org/10.1002/hipo.20796

Vann SD, Nelson AJD (2015) The mammillary bodies and memory: More than a hippocampal relay. In: Progress in Brain Research. Elsevier B.V., pp 163-185

Whelan S a., Dias WB, Thiruneelakantapillai L, et al (2010) Regulation of insulin receptor substrate 1 (IRS-1)/AKT kinase-mediated insulin signaling by O-linked ??-N-acetylglucosamine in 3T3-L1 adipocytes. J Biol Chem 285:5204-5211. https://doi.org/10.1074/jbc.M109.077818

Yamada M, Ohnishi H, Sano S I, et al (1997) Insulin receptor substrate (IRS)-1 and IRS-2 are tyrosinephosphorylated and associated with phosphatidylinositol 3-kinase in response to brain-derived 
neurotrophic factor in cultured cerebral cortical neurons. J Biol Chem. Nov 28;272(48):30334-9. doi: 10.1074/jbc.272.48.30334.

Yan Y, Dominguez S, Fisher DW, Dong H (2018) Sex differences in chronic stress responses and Alzheimer's disease. Neurobiol Stress. Mar 26;8:120-126. doi: 10.1016/j.ynstr.2018.03.002.

Yankelevitch-Yahav R, Franko M, Huly A, Doron R (2015) The Forced Swim Test as a Model of Depressive-like Behavior. J Vis Exp. https://doi.org/10.3791/52587

Yarchoan M, Toledo JB, Lee EB, et al (2014) Abnormal serine phosphorylation of insulin receptor substrate 1 is associated with tau pathology in Alzheimer's disease and tauopathies. Acta Neuropathol 128:679-689. https://doi.org/10.1007/s00401-014-1328-5

Yuan M, Meyer T, Benkowitz C, et al (2017) Somatostatin-positive interneurons in the dentate gyrus of mice provide local- and long-range septal synaptic inhibition. Elife 6:. https://doi.org/10.7554/eLife.21105

Zarei M, Patenaude B, Damoiseaux J, et al (2010) Combining shape and connectivity analysis: an MRI study of thalamic degeneration in Alzheimer's disease. Neuroimage 49:1-8. https://doi.org/10.1016/j.neuroimage.2009.09.001

Zou D, Chen L, Deng D, et al (2016) DREADD in Parvalbumin Interneurons of the Dentate Gyrus Modulates Anxiety, Social Interaction and Memory Extinction. Curr Mol Med 16:91-102. https://doi.org/10.2174/1566524016666151222150024

Addgene: AAV Purification. https://www.addgene.org/protocols/aav-purification-iodixanol-gradientultracentrifugation/. Accessed 14 Feb 2020 


\section{Figure Legends}

Figure 1. Experiment design. Bilateral injection of the viral particle (AAV-CMV-GFP, 4.94E+11 $\mathrm{GC} / \mathrm{mL}$ or AAV-CMV-shIRS1-EGFP,1.02E+10 GC/mL) (A) into the dorsal hippocampus (AP -4.36 mm, \pm ML $2.5 \mathrm{~mm}$, DV $-2.5 \mathrm{~mm}$ from bregma); $2 \mu \mathrm{L}$ of viral particle per hemisphere were injected (B). Behavioral tests started 33 days post-injection, and the animals were sacrificed at day 38 (C).

Figure 2. AAV shIRS1 impairs spatial memory. Time exploring the novel object in the Novel Object Recognition test (A). Time exploring the novel arm (B) and the number of entries to the novel arm (C) in the T maze test. Latency to cross the center of the arena for the first time in the Open Field test (D). Time spent immobile in the Forced Swim test (E). Data are expressed as discrimination index [(Time exploring novel - time exploring familiar) / total time exploring], presented as mean \pm whiskers at maximum and minimum ( $\mathrm{n}=7-8$ per condition) and analyzed using one-tailed Student t-test *p $<0.05$.

Figure 3. AAV DJ8 traces dorsal hippocampus efferents. Schematic representation of the projections from the dorsal hippocampus, where the viral particle was inoculated (A). Representative images of 3 animals (per condition) and 3 slices each of neuronal somas in the dorsal subiculum (DS) (B), and the polymorphic layer of the dentate gyrus (C); AAV labeled fibers in the medial septum (MS) (E) and supramammillary nucleus $(\mathrm{SuM})(\mathbf{F})$ via fornix (f) (D). Scale bar $=200 \mu \mathrm{m}(\mathrm{B})$. Scale bar $=100 \mu \mathrm{m}$ $(\mathrm{C}, \mathrm{D}, \mathrm{E}, \mathrm{F})$.

Figure 4. AAV DJ8 labels dorsal specific hippocampal neurons. Schematic representation of the dentate gyrus of the dorsal hippocampus (A). Percentage of double-labeled neurons (EGFP + neuronal marker), relative to the total number of neuronal marker-positive cells. Data are represented as the mean \pm SEM ( $\mathrm{n}=11)$. Representative images of 4-6 animals (per condition) and 3 slices each of EGFP (green) co-localizing with calretinin (CR) (C): Parvalbumin (PV) (D); and Somatostatin (SOM) (E) positive neurons in the polymorphic area (PoDG) of the dentate gyrus. Scale bar=100 $\mu \mathrm{m}$. Scale bar=20 $\mu \mathrm{m}$ (insets).

Figure 5. AAV labeled axons in the Medial Septum. Schematic representation of the Medial Septum (MS), vertical limb diagonal band (VDB), and horizontal limb diagonal band (HDB) (A). Representative images of 3 animals (per condition) and 3 slices each of showing AAV infected neuron projections colocalized with parvalbumin (PV) (B), cholinergic (ChAT) (C) and calbindin (CB) area (D). We observed no co-localization of AAV green fibers with calretinin (CR) positive neurons field (E). Scale bar=100 $\mu \mathrm{m}$. Scale bar $=20 \mu \mathrm{m}$ (insets).

Figure 6. Silencing effect of shIRS1 in the dorsal hippocampus. Schematic representation of the dentate gyrus of the dorsal hippocampus (A). Percentage of double-labeled neurons (IRS1+ EGFP), relative to the total number of EGFP-positive neurons (B). Representative images of IRS1, EGFP and colocalization of both labeling (merge) in the polymorphic area (PoDG) of control (C) and shIRS1 (D) animals. Data are represented as the mean \pm SEM $(n=10-11)$. Data were analyzed using one-tailed Student t-test $* * * p<0.0001$. Scale bar $=100 \mu \mathrm{m}$. Scale bar $=20 \mu \mathrm{m}$ (insets).

Figure 7. AAV-CMV-shIRS1-EGFP inoculation in the dorsal hippocampus results in lower synaptophysin positive labels in fibers reaching medial septum. Schematic representation of the Medial Septum (A). The number of contacts of synaptophysin (Synap) in EGFP-labeled fibers (B). Representative images of synaptophysin co-localization with AAV control (C) and EGFP-shIRS1 (D) fibers. Data are represented as the mean \pm SEM of the number of synaptophysin contacts with the green AAV labeling per $\mathrm{mm} 2$ of the fiber $(\mathrm{n}=3-4 ; 10$ fibers per animal). Data were analyzed using one-tailed Mann Whitney t test $* \mathrm{p}<0.05$. Scale bar $=10 \mu \mathrm{m}$. 
